intralobar lung sequester, as was confirmed with histologic examination of the resected left lower lobe. In retrospect, the benefit of preserving a small diseased segment of the lung seemed minor compared with the risk of infection and reoperation in case of necrosis. Therefore, we believe that resection of the affected lung segment remains the safest treatment option for symptomatic intralobar lung sequester.

\section{References}

1. Yamanaka A, Hirai T, Fujimoto T, Hase M, Noguchi M, Konishi F. Anomalous systemic arterial supply to normal basal segments of the left lower lobe. Ann Thorac Surg. 1999;68:332-8.

2. Baek WK, Cho J, Kim JT, Yoon YH, Kim KH, Lim HK, et al. Systemic arterial supply to normal basal segments of the left lower lobe along with the pulmonary artery: is lung resection warranted? J Thorac Cardiovasc Surg. 2006;131:742-3.

\title{
False-positive mediastinal lymph node activity on positron emission tomographic scan after adjuvant treatment of gynecologic malignancies
}

\author{
Bryan A. Whitson, MD, Sara J. Runge, BS, Shawn S. Groth, MD, \\ and Michael A. Maddaus, MD, Minneapolis, Minn
}

fter patients have undergone adjuvant treatment for gynecologic malignancies, newly positive mediastinal lymph nodes (LNs) on integrated 18-fluorodeoxyglucose (FDG) positron emission tomography (PET) and computed tomography (CT) images may suggest recurrent metastatic disease. We present the cases of 2 patients who underwent cytoreductive abdominal surgery and adjuvant chemoradiation for gynecologic malignancies whose subsequent follow-up surveillance FDG-PET/CT scan results revealed PET-positive mediastinal LNs. Neither patient had any previous thoracic disease, and prior FDG-PET results were negative.

\section{Clinical Summary}

\section{Patient 1}

A 57-year-old woman with stage IIIc endometrioid uterine adenocarcinoma underwent total abdominal hysterectomy, bilateral salpingo-oophorectomy, pelvic LN dissection, cytologic washings, and omentectomy. Final pathology demonstrated tumor invasion into the myometrium, positive estrogen and progesterone receptors on the tumor, and metastasis to 2 of 31 resected pelvic LNs. Three cycles of systemic chemotherapy (docetaxel and carboplatin), whole pelvic radiotherapy and brachytherapy, and 3 additional

From the University of Minnesota Department of Surgery, Section of Thoracic and Foregut Surgery, Minneapolis, Minn.

Received for publication Dec 4, 2006; accepted for publication Jan 5, 2007.

Address for reprints: Michael A. Maddaus, MD, Professor and Program Director, University of Minnesota Department of Surgery, MMC 207, 420 Delaware St SE, Minneapolis, MN 55455 (E-mail: madda001@umn.edu).

J Thorac Cardiovasc Surg 2007;133:1385-6

$0022-5223 / \$ 32.00$

Copyright () 2007 by The American Association for Thoracic Surgery doi:10.1016/j.jtcvs.2007.01.017

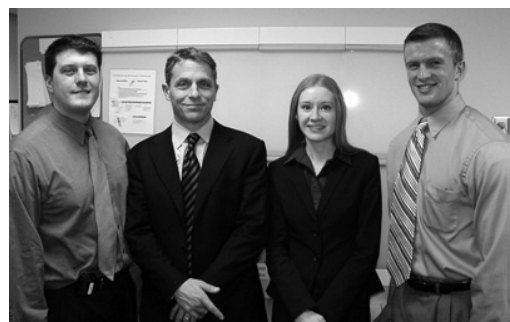

Drs Whitson, Maddaus, Runge, and Groth (left to right) cycles of chemotherapy (docetaxel and carboplatin) were given. An integrated FDG-PET/CT scan obtained 6 months after completing adjuvant treatment revealed newly positive perihilar and subcarinal LN, raising concerns of metastatic disease (Figure 1). Her carbohydrate antigen (CA)-125 level was $13 \mathrm{mg} / \mathrm{dL}$ (normal range, 0-30 mg/dL). Diagnostic mediastinoscopy (stations 3, 4R, and 7) was performed; the LN biopsy results demonstrated necrotic granulomas negative for fungi, mycobacteria, and malignancy. A repeat FDG-PET/CT scan 6 months later demonstrated resolution of all abnormal metabolic activity (Figure 1, C). Followup CA-125 levels have remained normal. Two years after the initial operation, she is alive without evidence of recurrent disease

\section{Patient 2}

A 61-year-old woman with stage Ic uterine carcinosarcoma underwent total abdominal hysterectomy, bilateral salpingo-oophorectomy, pelvic LN dissection, cytologic washings, and omentectomy. Final pathology demonstrated myometrial and angiolymphatic invasion. None of the pelvic LNs were positive $(0 / 25)$ for malignancy. She underwent 3 cycles of adjuvant chemotherapy (cisplatin and ifosfamide), whole pelvic radiotherapy, and 3 additional cycles of systemic chemotherapy (cisplatin and ifosfamide). An integrated FDG-PET/CT scan obtained 4 months into her chemoradiation treatment demonstrated newly positive perihilar and subcarinal LNs, raising concerns of metastatic disease. Diagnostic mediastinoscopy (stations $4 \mathrm{R}$ and 7) was performed. The LN biopsy results demonstrated normal LN with no pathologic disease and were negative for fungi and mycobacteria. Follow-up FDG-PET/CT imaging 9 months later demonstrated resolution of the abnormal mediastinal metabolic activity. Her CA-125 level was $45 \mathrm{mg} / \mathrm{dL}$ at the time of mediastinoscopy and $12 \mathrm{mg} / \mathrm{dL}$ at the time of repeat imaging. Fourteen months after the initial operation, she is without evidence of recurrent disease. 

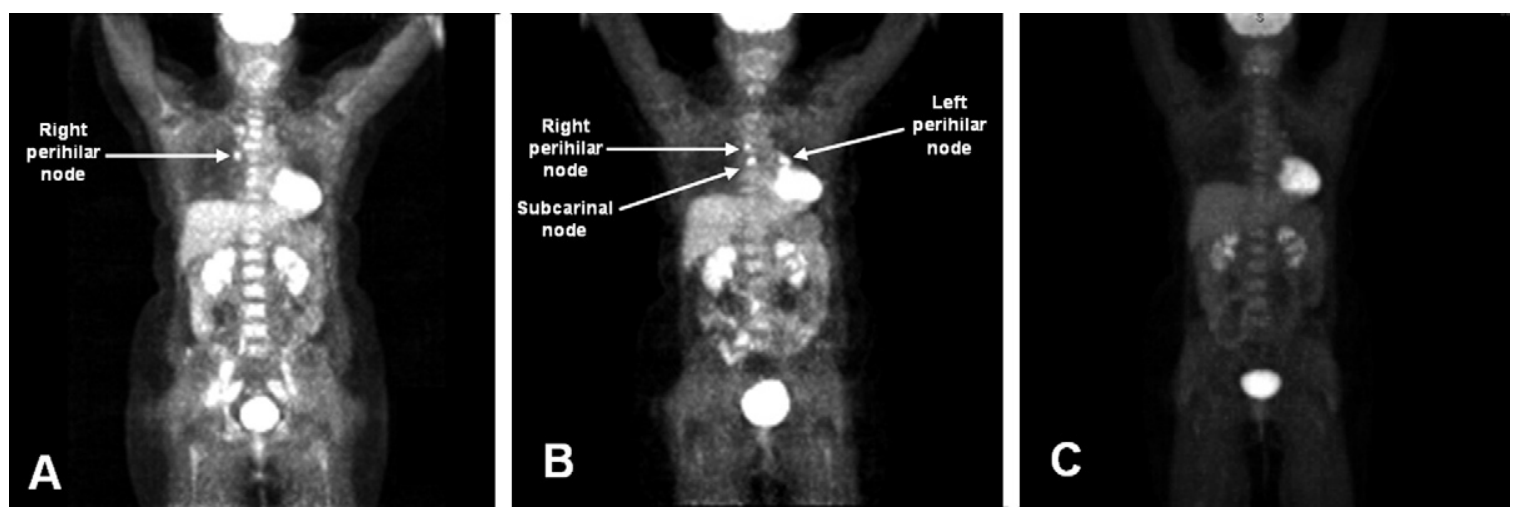

Figure 1. Selected images from serial integrated FDG-PET/CT scans of patient 1. A, This image, obtained after the gynecologic surgery, demonstrates a calcified right perihilar LN with a maximum standard uptake value (SUV) of 4.0, believed to be consistent with granulomatous changes. $B$, This image, obtained after the end of adjuvant chemotherapy and radiation treatment (6 months after image A), demonstrates that the same right perihilar LN, still the same size $(12 \mathrm{~mm})$, now has a maximum SUV of 4.6. A subcarinal LN demonstrates new metabolic activity, with a maximum SUV of 5.2. A left perihilar $L N$ has increased in size $(26 \mathrm{~mm})$ with new metabolic activity and a maximum SUV of 5.1. C, This image, obtained 6 months after the image in (B), demonstrates no areas of abnormal metabolic activity.

\section{Discussion}

Because of the fusion images of newer integrated PET/CT machines, CT and PET scans are increasingly used to screen for recurrent disease in patients with cancer, individually, serially, or concomitantly. When FDG-PET is used for preoperative staging, false-positive results can occur. In Cerfolio and colleagues' study ${ }^{1}$ of 400 patients with non-small cell lung cancer (NSCLC), the false-positive rate was as high as $76 \%$ for preoperative $\mathrm{N} 2$ disease. They found that PET-positive LNs at station $4 \mathrm{R}, 4 \mathrm{~L}$, and 7 were most likely to be false-positives (as in our 2 patients). When FDG-PET scans are used as a postoperative surveillance tool in patients with NSCLC, false-positives can also occur, but at a much lower rate. According to another study, FDG-PET scans, when used for recurrence surveillance at a median of 19 months after primary therapy in patients with NSCLC, had a sensitivity of $93 \%$ and a specificity of $89 \%$, with only $2.7 \%$ false-positives $(2 / 73) .^{2}$

In 2 studies of gynecologic malignancies (uterine and cervical carcinomas), FDG-PET scans used for surveillance after treatment had a sensitivity of $90 \%$ to $96 \%$ and a specificity of $70 \%$ to $78 \%$. 3,4 The false-positive rate ranged from $10.7 \%$ to $65 \%$ for thoracic LN lesions. The true mediastinal or hilar $\mathrm{LN}$ recurrence rate was $26.5 \%$ to $46.2 \%$. However, the false-positive rate for hilar and mediastinal LNs was as high as $73.5 \% .^{4}$ Neither of these gynecologic studies routinely used serial FDG-PET examinations.

To our knowledge, no other specific cases have been reported of delayed metabolic activation of mediastinal LNs causing falsepositive images after treatment for gynecologic malignancies. Pos- sible explanations for false-positive LNs include infection, benign inflammatory processes, and residual inflammation from local treatment effects. ${ }^{3-5}$ In our 2 patients, the mediastinal LNs were negative for fungi, bacteria, and malignancy, none of which were manifest in the radiation field after adjuvant therapy.

New FGD-PET-positive mediastinal LNs manifesting after treatment of gynecologic malignancies raise concerns for metastatic disease. Although false-positives can occur, tissue biopsies must be obtained to definitively exclude metastatic disease.

The authors thank Mary Knatterud, PhD, for her editorial assistance.

\section{References}

1. Cerfolio RJ, Ojha B, Bryant AS, Bass CS, Bartalucci AA, Mountz JM. The role of FDG-PET scan in staging patients with nonsmall cell carcinoma. Ann Thorac Surg. 2003;76:861-6.

2. Hellwig D, Groschel A, Graeter TP, Hellwig AP, Nestle U, Schafers HJ, et al. Diagnostic performance and prognostic impact of FDG-PET in suspected recurrence of surgically treated non-small cell lung cancer. Eur J Nucl Med Mol Imaging. 2006;33:13-21.

3. Belhocine T, De Barsy C, Hustinx R, Willems-Foidart J. Usefulness of (18)F-FDG PET in the post-therapy surveillance of endometrial carcinoma. Eur J Nucl Med Mol Imaging. 2002;29:1132-9.

4. Ryu SY, Kim MH, Choi SC, Choi CW, Lee KH. Detection of early recurrence with 18 F-FDG PET in patients with cervical cancer. $J$ Nucl Med. 2003;44:347-52.

5. Yen TC, Lai CH. Positron emission tomography in gynecologic cancer. Semin Nucl Med. 2006;36:93-104. 